\title{
TRANSIENT RELAPSING ENOPHTHALMOS OF SYMPATHETIC ORIGIN.
}

\author{
JosÉ DE J. GonzÁLez, M. D. \\ LEÓN, MEXICO.
}

\begin{abstract}
The case here reported differs materially from the conditions of enophthalmos described in the text-books in the striking features of the attacks and the association of enophthalmos with pregnancy. Translated from the Spanish by M. Uribe-Troncoso, M. D.
\end{abstract}

The opportunity has been offered to me for observing a very interesting case of transient enophthalmos, with some unusual features, making it worth recording.

Mrs. C., 25 years old, from Aruascalientes, Mexico, well buiit and strong, has always been in good health. No history of neuropathic disease could be elicited. She was married a year ago and almost immediately showed signs of pregnancy. On the third or fourth month, without any apparent cause, when dressing her hair before a mirror, she felt suddenly that her left eye sank in the orbit, a fact that she could immediately verify at the mirror; the eye appearing smaller and sunken and the palpebral fissure narrower. Moreover, the skin on both lids became entirely white and bloodless. She was very much frightened, and went to her husband and other persons in the family, who were able to verify these symptoms. A sensation of torpidity and difficulty of moving the lids and the eyeball were present.

After fifteen minutes of this paroxysm the skin of the lids became colored, the palpebral aperture widened and the eye came forward. The patient thought all was over, but two or three weeks afterwards the condition returned and then reappeared several times at irregular intervals.

The family physician sent the patient to me. I had the good fortune of witnessing one of the seizures. The syndrome began with marked paleness of the left side of the face, specially noticeable in the lids and conjunctiva, which were completely bloodless. A few minutes later the patient had the sensation of sinking of the eyeball; and an examination revealed a narrow- ing of the palpebral fissure, ptosis of $20^{\circ}$, measured with the perimeter, and a recession of the eyeball in the orbit of about five millimeters.

The pupil was smaller than that of the other eye. The patient was able to lift the upper lid only to a very limited degree. The brow on the side of the ptosis was not drawn up, and there were no wrinkles on the forehead, as is the case when there is a palsy of the levator. On the contrary, the brow was lower and the wrinkles were formed on the lids themselves, as in the pseudoparalytic ptosis of hysteria, which is due to a contraction of the orbicularis.

The movements of the eyeball were difficult and delayed, but the excursions were normal. The fundus showed no signs of abnormality. Intraocular tension was diminished.

After fifteen or twenty minutes the skin of the lids recovered its color, the palpebral aperture widened, the eyeball advanced in the orbit and the whole paroxysm ended, only leaving the patient much frightened and greatly depressed. A careful examination made afterwards failed to detect any abnormality in the eyes. Refraction was normal; the mobility of the pupil and eyeball normal. Vision $=1$, in both eyes. Visual fields and color vision normal; as were also the conjunctival and corneal reflexes.

The diagnosis in this case is not difficult; we are dealing with a transient, relapsing enophthalmos, attended with ptosis, myosis, hypotension and spasm of the cutaneous and mucous vessels, due to a nervous disturbance; the motor, sensitive and angiospastic symptoms being characteristic. But to what part of the nervous system must they 
be ascribed? What is the anatomic diagnosis?

There are paradoxic symptoms which are difficult to interpret if we are going to be guided only by the physiologic experimentation. In fact, the intense paleness of the lids and conjunctiva on the left side alone, the narrowing of the palpebral aperture, the diminished intraocular tension, the enophthalmos and myosis, are all symptoms of a disturbed condition of the sympathetic nerve on one side.

According to physiologic investigations, paralysis of the sympathetic produces:

(a) Retraction of the eyeball due to paralysis of the smooth muscular fibres of Tenon's capsule.

(b) Narrowing of the palpebral aperture due to paralysis of the smooth muscles of the lids, which cannot balance the stronger and opposing action of the orbicularis.

(c) Myosis, which may be due either to a paralysis of the dilatator iridis or, as nthers point out, to the suppression of the inhibitory action of the sympathetic upon the sphincter of the iris. sion.

(d) Diminished intraocular ten-

(e) Dilatation of the blood vessels, showing itself by redness of the skin, of the conjunctiva, ear, auricle, etc.

However, in my patient, instead of the last symptom there was the opposite condition: a marked angiospasm of the skin and the conjunctiva, undoubtedly due to a stimulation of the sympathetic. How can we explain these contradictory symptoms? In the first place, the physiology of the sympathetic is still surrounded by darkness, and the pathologic physiology sometimes produces true dissections; that is to say, separates the functions of one organ in different ways. It is a well known fact that the cervical sympathetic gives to the vessels, not only vasoconstrictive fibres but also vasodilator; whose antagonistic effect maintains the vascular balance. If we suppose that the paralysis was confined only to the vasodilators, leaving intact the action of the opposite fibres, then the predominant action of the former will produce the paleness of the skin and conjunctiva; symptoms not really of stimulation, but only of lack of the opposite action or tonus.

In regard to the cattse of this transient paralysis of the sympathetic, it is necessary to suppose in the absence of any other cause that it was due to pregnancy. The influence of pregnancy upon some trophic disturbances, which are due to the sympathetic, is now well almitted, i. e., the so called cutaneous syndrome, pigmentation around the nipples, the pigmentary line on the $a b-$ domen, pigmentation of the face, etc. We are probably not far away from the real fact in considering this relapsing sympathetic paralysis as due to pregnancy.

What is more difficult to ascertain is, if it is due to a purely reflex action; or to the toxins arising from pregnancy and circulating through the system. In favor of a reflex action is the rapidity of the outbreak and its transient character. But an influence of the autotoxins upon the ganglion cells of the sympathetic cannot be excluded. An clective action may be produced just the same as strychnin has a selective convulsive action, morphin an analgesic effect and curare only acts upon the motor terminations of the nerves. 